\title{
THE PHYSICS OF SEMICONDUCTOR SURFACES
}

$S^{\mathrm{U}}$ URFACE physics has not usually been thought of as a particularly exact science. The experimental difficulties in obtaining meaningful measurements under well-controlled chemical conditions, and the theoretical difficulties of proceeding beyond a mere thermodynamical description to an atomistic, quantum-mechanical one, have alike tended to keep surface physics on the by-ways of science. It is, nevertheless, a field which is of growing importance, in physical science, in biological science and in technology. A measure of the progress recently gained may be gathered from the scope of a conference on the "Physics of Semiconductor Surfaces", held in Philadelphia last June. The conference, which was sponsored by the Office of Naval Research, Lincoln Laboratories, and the University of Pennsylvania, included representatives from Canada, France, Germany, Great Britain and the Netherlands, as well as the United States. Although much of the conference was devoted to the surfaces of silicon and germanium, there were contributions from chemists in the fields of oxidation and catalysis. The papers presented at this conference are to be published in book form in the near future by the University of Pennsylvania Press. In the meantime, a brief review of the conference, and a few comments on one or two of the papers-picked almost at random-will be given in this short account.

Two things have helped to promote progress in surface physics during the past ten years. One is the development of techniques for carrying out experiments in ultra-high vacuum. An atomically clean surface, kept in a vacuum of $10^{-10} \mathrm{~mm}$. of mercury, should have a monolayer adsorption time of several hours-long enough to perform detailed measurements on it. The other stimulus-and the one more immediately responsible for the promotion of the Philadelphia conference-has come from the invention of the transistor and, with it, the availability of germanium and silicon in a state of very high purity. This means that one can now carry out experiments on a surface system in which it is possible to change the electrochemical potentials of holes and electrons at will by known amounts. It also means that, because the surface space-charge region extends relatively far into the semiconductor, the several contributions to the overall variation of electrostatic potential across the interface may each be determined quantitatively. The ability to distinguish the different parts of the surface dipole has hitherto been lacking in the analysis of solid-gas surface systems, although there is a rough analogue in electrochemistry, in the measurement of zeta potentials. The material presented at the conference affords a good illustration of these developments.

Many of the papers dealt with surfaces of germanium and silicon, as prepared by etching and maintaining in some gaseous environment. Here the physicist can make detailed measurements on the positions of electronic energy-levels associated with the surface, and of transition probabilities between these levels and the interior of the semiconductor. The physicist distinguishes between 'fast' and 'slow' statos. The 'fast' states, characterized by relaxation times of less than a microsecond, must be associated with the actual germanium or silicon surface; the 'slow' states have relaxation times of the order of a second or longer and, in the present state of knowledge, are imagined to be associated with the oxide laver. Several papers gave quantitative information on the distribution of the 'fast' states in the forbidden energy gap, and on the capture cross-sections for these states. The most valuable experimental tools here are the effects of applying a high electric field in a direction normal to the surface, and of illuminating it. Other papers dealt with the 'slow' states and the probable connexion between these states and $(1 / f)$ noise.

All these experiments suffer from the drawback that the chemical constitution of the surface region is almost completely unknown, and largely uncontrollable. Many workers have therefore tackled the problem of carrying out similar measurements on an atomically clean surface, and then allowing various impurities to be deposited upon it, so that the effect of each one may be studied. P. Handler reviewed the experimental results obtained by the Farnsworth technique of ion bombardment and annealing in ultra-high vacuum. The most outstanding conclusion here is the unexpectedly high surface conductivity found after the surface has been prepared in this way. It was generally agreed that the Farnsworth surface is $p$-type, becoming less so as the surface is exposed to reasonable amounts of oxygen. There is quite good agreement between the findings of the various research teams working in this field. These facts, if they do indeed refer to the properties of an atomically clean surface, are of the highest importance, because this is the only case so far for which it has been possible to give anything approaching a respectable theoretical discussion. The theory of surface states was discussed by C. Herring, who directed attention to the Tamm and Shockley states associated with a perfect semiconductorvacuum interface, and to the various kinds of level arising from surface impurities and imperfections.

Turning to matters of chemical interest, there was an excellent series of papers on the electronic aspects of catalysis. K. Hauffe discussed his work on the effects of the surface space-charge region on catalysis at semiconductor surfaces. G. M. Schwab presented a solection of experimental results, some of them obtained with germanium-which, he said, is not a very good catalyst, but illustrates the consequences of the theory quite nicely. The final session of the conference was devoted to surface oxidation, including a discussion of nucleation effects, by N. Cabrera, and several papers on the surface oxidation of germanium under various conditions.

The general level of the papers and discussions was exceptionally high, and most people seemed to feel that both the formal and the informal activities at the conference were profitable and stimulating. Surface physics is in a very exciting state of growth at the moment, and, if the purist views the current heavy emphasis on silicon and germanium with a somewhat jaundiced eye, he may take comfort in the expectation that the techniques and understanding developed with these materials will be of direct usefulness in the study of other surface systems.

C. G. B. Garrett 